\title{
Perspectives in the use of peptidomics in ham
}

5

6

Marta Gallego, Leticia Mora, and Fidel Toldrá*

7

8

9 Instituto de Agroquímica y Tecnología de Alimentos (CSIC), Avenue Agustín Escardino 7, 46980, Paterna (Valencia), Spain

11

12

13

14

15

16

17

18

$19{ }^{*}$ Corresponding author: Tel: +34963900022 ext.2112; fax: +34963636301.

20

$21 \quad$ E-mail address: ftoldra@iata.csic.es

22

23

24 Keywords: Dry-cured ham, peptidomics, peptides, bioactive peptides, mass spectrometry.

25 Abstract 
26 The use of proteomics in food science has permitted both a better characterisation of the

27 food products and the control of their final quality despite the fact that food systems are

28 under continuous changes during processing and consumption. Ham is a very popular

29 meat product frequently consumed in Mediterranean countries after a dry-curing

30 processing that results in the generation of large amounts of peptides that contribute to its

31 texture, flavour, and final quality. Proteolysis is the main biochemical mechanism

32 occurring throughout the dry-cured ham processing and a better knowledge of this

33 phenomenon is essential to give an extra-value to the product and produce regular batches.

34 In this regard, peptidomics has become an important tool for the characterisation of dry-

35 cured hams in order to identify and quantify bioactive peptides and potential biomarkers,

36 study their stability under processing conditions or gastrointestinal digestion, and search

37 for post-translational modifications that could affect the quality of dry-cured hams. This

38 manuscript is reviewing from the first uses of comparative proteomics in dry-cured ham

39 approaches to the most modern peptidomic studies and their contribution to the advance

40 in meat science.

41 


\section{Introduction}

43 The application of proteomic approaches in food permits better characterisation of the

44 product as well as improvement and control of its final quality. In fact, due to the fact that food can be considered a biological system that is under continuous changes, studies about protein degradation or post-translational modifications have been in the focus since the last decade. Advances in both mass spectrometry instruments and experimental approaches have permitted the evolution from 'bottom-up' approaches, mainly used in proteomics, to more sophisticated 'top-down' peptidomic experiments that include the difficult data analysis resulting from unspecific hydrolysis.

Peptidomics has been used to follow proteolysis in natural and complex food processes such as curing or fermentation, characterise the effect of processing, follow the simulated gastrointestinal digestion, search for potential peptides to be used as biomarkers, or detect characteristic peptides to differentiate between genotypes and products [1-4].

Ham is a very popular product that is frequently consumed in Mediterranean countries after dry-curing, a processing step that results in the generation of different peptides that contribute to its texture, flavour, and final quality. One of the main biochemical mechanisms occurring in the processing of dry-cured ham is proteolysis, which mainly occurs through the action of endogenous muscle peptidases. The control of proteolysis is of primary importance in order to produce a consistent, regular, and high quality product, so a better knowledge of this phenomenon is essential to give an extra-value and produce regular batches [5].

63 The use of peptidomic approaches in dry-cured ham has contributed to an advancement

64 in the meat science field through the knowledge of the generated peptide sequences, which have resulted in an added-value of the product due to the presence of natural bioactive peptides and markers generated during curing or final quality of the product. 
67 In this paper, a review from the first uses of comparative proteomics in dry-cured ham

68 approaches to the most modern peptidomic studies and their contribution to the

69 advancement in meat science has been done.

\section{Prospects and limitations in the use of proteomics in studying naturally generated}

\section{peptides}

73 Proteolysis has been identified as the key phenomenon responsible for the generation of peptides during food processing. Techniques like SDS-PAGE, 2D gel electrophoresis, free solution conjugate electrophoresis (FSCE), SEC and HPLC with reversed phase, hydrophilic interaction liquid chromatography (HILIC) or ion exchange chromatography (IEC) have been frequently used in studies based on the comparison of proteomes $[6,7]$.

These techniques combined with single MS revealed information about the breakdown of proteins that occurs post-mortem and during processing of meat. These approaches also elucidated the importance of the generation of peptides derived from the intense action of muscular enzymes.

Main 'bottom-up' approach used in dry-cured ham studies is the Peptide Mass Fingerprint (PMF), which is useful for the follow-up of protein degradation along different times and/or between different samples. In this regard, the combination of 2D SDS-PAGE and MALDI-TOF MS analysis allowed the study of changes in myofibrillar and sarcoplasmic fractions of raw and dry-cured hams at different ripening times [6]. Thus, the progressive

87 degradation of actin, tropomyosin and myosin light chains as well as certain sarcoplasmic 88 proteins was observed during the curing of ham in the 2D gels. In this study, main bands were digested using trypsin and subsequently analysed by MALDI-TOF MS. A similar proteomic approach was used to compare the proteomic maps of two muscles, biceps

91 femoris and semimembranosus in dry-cured hams, showing that the methodology is very 
92 useful for muscular differentiation [8]. A PMF approach was also applied to evaluate the

93 changes occurring in sarcoplasmic proteins after proteolysis and during the ripening of

94 meat products [9]. In that study, the separation of proteins by 2D AUT-PAGE/SDS

95 electrophoresis, which combines an acetic acid-urea-triton polyacrylamide gel with the

96 classic SDS gel, was used together with a MALDI-TOF mass spectrometer for the

97 identification of 13 sarcoplasmic proteins. Although this technique showed a higher

98 resolution in comparison to standard 2D IPG/SDS-PAGE as well as an easier

99 interpretation of the 2D maps, current tendencies are focused on non-destructive and fast

100 techniques. In this sense, spectroscopic high-resolution magic angle spinning (HRMAS)

101 NMR [10] is a complementary technique for proteomic tissue characterisation in which

102 different spectral peaks are assigned to different protein components. Also the non-

103 destructive quantitative magnetisation transfer magnetic resonance imaging (qMT-MRI)

104 methodology can be employed to characterise dry-cured ham tissues differing in

105 anatomical positions and processing protocols using the binary-spin-bath (BSB) model

106 [11]. Recently, a combination of the immunochemical and MS methodologies SDS-

107 PAGE, Western blotting and blot overlay assays together with an linear trap quadrupole

108 (LTQ) Orbitrap mass spectrometer were used to evaluate the changes ocurring in actin

109 and myosin heavy chain proteins in the biceps femoris muscle during the processing of

110 three Italian dry-cured hams [12]. Finally, some studies combined qualitative and

111 quantitative proteomics using MS in tandem for proteome characterisation.

112 Quantification using a label-free approach has been applied to determine the changes in

113 water-soluble proteins at different processing times [13] and to evaluate the effect of a

114 pressure treatment applied during the salting step on the processing of dry-cured hams

115 [14]. In this regard, MS quantitative methods have been proven to be precise and effective 
116 alternatives to traditional techniques based on gel electrophoresis that present low

117 specificity, limited dynamic range and inaccurate identification of overlapping proteins.

118 However, all these studies were focused on the proteolysis phenomena from the point of

119 view of protein degradation under different conditions and different samples but no

120 specific sequences of the generated peptides were described. In this sense, a better

121 knowledge of the generation of peptides during the different food processes and their

122 specific sequences is essential to be able to confer an added-value to the final product due

123 to their bioactive properties in the human body or as instruments for quality control. The

124 identification of naturally generated peptides requires the analytical and experimental

125 adaptation of previously used strategies for the identification of proteins such as tandem

126 MS and modern bioinformatics tools; however, different challenges make the

127 identification of natural peptides very difficult. The characteristics of these peptides could

128 be very different depending on the degree of protein proteolysis. The classic PMF

129 approach, which is very frequent in proteomics for the identification of proteins, is not

130 useful in this case because these peptides cannot be trypsin digested due to their small

131 size. Thus, the analysis of di- and tripeptides are sometimes in the limit of some standard

132 MS techniques, requiring an extra effort for their unequivocal identification. On the other

133 hand, longer peptides face up the impossibility of controlling the hydrolysis, resulting in

134 a complex mixture of peptides derived from different proteins with unspecific cleavage

135 sites [15].

136

\section{3. Peptidomics in the characterisation of dry-cured ham}

138 Peptidomics has become an important tool for the characterisation of dry-cured ham, as

139 this complex matrix contains a great variety of peptides naturally generated due to the

140 action of endogenous proteolytic enzymes during the long processing of this product. This 
141 mixture of peptides shows a wide range of sizes and non-specific cleavage sites, which

142 requires different pretreatments and chromatographic steps such as SEC or HPLC to

143 avoid interferences and isolate peptides prior to their identification and quantification by

144 MS analysis [16].

\section{$145 \quad 3.1$ Identification of peptides}

146 The use of tandem MS techniques allows a fast and reliable identification of the peptides

147 generated from the degradation of sarcoplasmic and myofibrillar proteins during the dry-

148 cured ham processing. For that, the obtained MS/MS spectra are matched with theoretical

149 peptide sequences contained in databases in order to elucidate the specific sequences. So,

150 numerous peptides derived from myofibrillar proteins such as actin [17], myosin light

151 chain $[2,18,19]$, troponin T [20,21], titin [18,22], LIM domain-binding protein 3 [23], and

152 myozenin [21], as well as peptides from sarcoplasmic proteins such as creatine kinase

153 [24], glycolytic enzymes [21,25], myoglobin [26], and ubiquitin [27] have been identified

154 in Spanish dry-cured ham using mainly MALDI-TOF MS and ESI-LC-Q/TOF MS. Table

1551 reports the number of peptides and their proteins of origin identified in Spanish dry-

156 cured ham. Several small peptides were also identified in Chinese dry-cured Jinhua ham

157 using MS in tandem, but their proteins of origin were not elucidated [28]. These studies

158 contribute to the characterisation of peptides in order to understand the role of muscle

159 enzymes in the intense proteolysis that happens during ham processing, which would be

160 affected by the raw material and processing conditions. Sarcoplasmic and myofibrillar

161 proteins are hydrolysed by endopeptidases, mainly calpains and cathepsins, which result

162 in the formation of longer polypeptides and changes in texture of dry-cured hams. Then,

163 polypeptides are degraded by exopeptidases including tri- and di-peptidyl peptidases,

164 dipeptidasas, aminopeptidases, and carboxypeptidases, generating small peptides and free

165 amino acids that contribute to the characteristic sensory properties of the product [29,30]. 
166 In this regard, certain dipeptides and free amino acids have been described to influence

167 flavour development in Spanish Serrano dry-cured hams [31], whereas two umami taste 168 peptides CCNKSV and AHSVRFY were identified from Chinese Jinhua ham and Italian

169 Parma ham, respectively [32].

\section{$170 \quad 3.2$ Quantification of peptides}

171 Peptidomic approaches usually include the absolute or relative quantification of peptides

172 by using labelling or label-free methodologies. The relative quantification is based on the comparison of peptide amounts among two or more samples. Labelled methods provide

174 the most accurate quantitative values, but they require complex experimental protocols

175 and expensive isotope labels. On the other hand, label-free methodologies allow a simple,

176 reliable, versatile, and cost-effective quantification, either on the basis of peak intensity

177 measurements or spectral counting. In quantification based on peak intensity, the signal

178 intensity measurements of extracted ion chromatograms correlate to the concentration of

179 that particular peptide, allowing a precise and accurate evaluation of changes in

180 abundance between samples [33, 34]. This label-free MS approach has been used to

181 identify and quantify the peptides naturally generated during the ham dry-curing process,

182 and study their evolution at different processing times [2].

183 Absolute quantification measures the total levels of a given peptide in the sample, either

184 by using label or label-free approaches. Among them, MRM has proven to be the most 185 suitable methodology, with a wide linear range. MRM is a highly sensitive and selective 186 method of targeted MS that generally employs tandem quadrupoles for the detection and 187 quantification of small and low abundance peptides in complex mixtures. So, the first 188 quadrupole (Q) act as filter to specifically select certain $m / z$ values of peptide ion, the 189 second Q serves as collision cell for ion fragmentation, and the third Q filters a specific 190 fragment ion of the peptide for its detection. Such precursor/fragment ion pairs 
191 (transitions) are monitored over time and the integration of their chromatographic peaks

192 allows the quantification of the target peptide $[35,36]$. However, there are several

193 challenges in the analysis due the complexity of the samples especially when peptides

194 have been naturally generated by unspecific enzymes or during processing conditions, as

195 well as in the optimisation of the MS parameters for an accurate and sensitive

196 quantification without interferences from other peptides or signal suppression [37]. MRM

197 methodologies have been recently used to quantify several peptides and amino acids

198 generated during the ripening of dry-cured hams [38,39] as well as to evaluate the

199 degradation of added microbial transglutaminase during the maturing process of dry-

200 cured ham through the use of isotope labelled peptide standards [40].

201 4. Specific applications of peptidomics in dry-cured ham

202 The applications of peptidomics include the characterisation of peptides in order to

203 identify and quantify bioactive peptides and potential biomarkers, study their stability

204 under processing conditions or gastrointestinal digestion, and search for post-translational

205 modifications in peptides that can affect the quality of dry-cured hams (Figure 1).

$206 \quad 4.1$ Discovery and bioavailability study of bioactive peptides

207 Dry-cured ham has been described as a good source of bioactive peptides showing 208 angiotensin converting enzyme (ACE) inhibition as well as antioxidant, antimicrobial, 209 and dipeptidyl peptidase IV (DPP IV) inhibitory activities. The discovery, bioavailability 210 and monitoring of bioactive peptides in complex food matrices such as dry-cured hams

211 usually require empirical strategies that involve the use of high-resolution separation

212 techniques, in vitro assays to detect the most active fractions, identification of peptides

213 by MS, selection and synthesis of potential bioactive peptides, and confirmation of their

214 bioactivity by in vitro or in vivo assays. Moreover, the combination of this traditional

215 workflow with bioinformatics tools or in silico analysis permits to obtain a large amount 
216 of data regarding the chemical structure, activity, enzyme-peptide interactions, and

217 availability of bioactive peptides [41].

218 Focusing on ACE inhibitory activity, water soluble peptide extracts from Spanish dry-

219 cured hams have been reported to exhibit up to $97.7 \%$ of in vitro ACE inhibition in some

220 fractions obtained after SEC $[42,43]$. Several peptides responsible for this activity have

221 been identified through MS analysis [44,45], reporting some potent ACE inhibitory peptides such as AAPLAP, KPVAAP and KAAAAP. In Parma dry-cured hams, a hybrid in silico/in vitro tandem approach was employed for the identification of strong inhibitory peptides such as LGL and SFVTT in fractions obtained after in vitro gastrointestinal digestion [46]. On the other hand, a study focused on the characterisation of the peptide profiles in Spanish Teruel, Italian Parma, and Belgian dry-cured hams evidenced the presence of ACE inhibitory and antioxidant peptides, showing higher ACE inhibition values in the Spanish ham probably due to its longer curing time [47]. The sequences of some ACE inhibitory peptides and their $\mathrm{IC}_{50}$ values (peptide concentration that inhibits $50 \%$ of ACE activity in the reaction mixture) are shown in Table 2.

231 Dry-cured hams have also been studied as a source of antioxidant peptides. In Spanish 232 dry-cured hams, the antioxidant activity of water soluble extracts has been evaluated and 233 the sequences of some of these antioxidant peptides have been identified by MS $[42,48]$.

234 In this regard, the peptide SNAAC has been the most potent antioxidant identified to date, 235 showing an $\mathrm{IC}_{50}$ value of $75.2 \mu \mathrm{M}$ in DPPH radical scavenging assay and $205 \mu \mathrm{M}$ in 236 ferric-reducing power analysis [49]. Concerning Chinese hams, the peptide GKFNV 237 showed the highest activity in Jinhua ham extracts [50,55], whereas DLEE would be one 238 of the main peptides playing a key role in the antioxidant activity of Xuanwei hams [51]. 239 Table 2 shows some of the antioxidant peptides identified in dry-cured hams and their 240 activity values. 
241 Regarding other bioactivities, several naturally generated peptides from Spanish dry-

242 cured ham have been described to have antimicrobial action against the pathogenic

243 Listeria monocytogenes, being RHGYM the most effective [52]. Also some dry-cured

244 ham peptides and dipeptides such as AAATP and KA have been reported to exert DPP

245 IV inhibitory activity and thus could be act as a natural therapeutic approach for the

246 management of type 2 diabetes [53]. Some examples of the identified peptides showing

247 antilisterial and DPP IV inhibitory activities are shown in Table 2.

248 Peptidomics has also constituted an indispensable tool to study the bioavailability of

249 bioactive peptides in order to assure that the active form reaches the target site to exert its

250 effect. The use of MS-based techniques has permitted to evaluate possible modifications

251 of the structure and activity of bioactive peptides that can occur during food processing,

252 gastrointestinal digestion and transport in the organism. For that purpose, MS analyses

253 are usually combined with computational tools and studies in cellular and animal models

254 [41]. Several studies have evaluated the generation of novel bioactive peptides after

255 simulated gastrointestinal digestion $[50,56]$ as well as the stability of ACE inhibitory and

256 antioxidant peptides against in vitro digestion and different processing/storage conditions

257 such as temperature, salt, $\mathrm{pH}$, and light exposure $[39,44,57,58]$. Furthermore, a recent

258 study based on a MRM-based methodology was able to quantify the amount of the

259 antioxidant peptide AEEEYPDL that was 0.148 fg per $\mathrm{g}$ of Spanish dry-cured ham [39],

260 which is crucial to evaluate the bioavailability and potential effect of the peptide in the

261 organism.

262 Cellular models provide useful information about intestinal absorption mechanisms and bioavailability of peptides. So, the transepithelial transport of several ACE inhibitory

264 peptides identified in Spanish dry-cured hams was studied through a Caco-2 cell

265 monolayer, evidencing the absorption and generation of novel ACE inhibitory peptide 
266 fragments during the process by MS techniques [59]. Nevertheless, in vivo studies are

267 needed to demonstrate the efficacy and physiological effects of bioactive peptides in the 268 organism. The potential antihypertensive action of dry-cured ham peptides have been

269 investigated by measuring changes in systolic blood pressure (SBP) in spontaneously 270 hypertensive rats (SHR). In this regard, the administration of Spanish ham extracts to 271 SHR showed a maximum decrease in SBP of $38.38 \mathrm{mmHg}$ after 8-hour ingestion [42], 272 being AAATP the most potent antihypertensive peptide $(-25.62 \mathrm{mmHg}$ at $8 \mathrm{~h})$ [45]. 273 Iberian dry-cured ham extracts decreased SBP by $12 \mathrm{mmHg}$ at $8 \mathrm{~h}$ in SHR, and the MS 274 analysis revealed the presence of large amounts of peptides containing the ACE inhibitory 275 fragments PPK, PAP and APP repeated in their sequences [43]. Finally, two recent human 276 studies have reported that the regular consumption of bioactive peptides contained in dry277 cured hams impair beneficial effects on cardiovascular health $[60,61]$.

\section{$278 \quad 4.2$ Biomarker search}

279 In general, dry-cured hams require extensive ripening-drying times that last up to 24 280 months or even longer in some cases. Long times of curing are related to a more intense 281 proteolysis and higher generation of flavour and aroma compounds, which principally 282 determine the quality and economic value of the product. Peptidomics can contribute to 283 process control by determining some peptides as markers of the time of processing, in order to prevent mislabeling and fraudulent activities resulting in over-valued products. In this respect, some peptides generated from LIM domain-binding protein 3 and 286 ubiquitin protein were suggested as potential markers to estimate and control the 287 processing time of Spanish dry-cured hams [23,27]. Moreover, four peptides derived from titin protein were identified by ESI- LC-Q/TOF MS as potential biomarkers of a minimum processing time of 9 months, and complementarily, MALDI-TOF MS could be employed as a fast and easier technique to check the presence of these peptide markers [22]. 
291 Differences obtained in the relative quantification of generated peptides during the

292 processing of dry-cured hams permitted to determine the main peptides and proteins

293 responsible for differences between dry-cured ham samples. So, peptides derived from

294 myosin light chain 1 protein were the most influential at the end of the dry-cured ham

295 processing, and specifically peptides APAPAPAPPKEEKI and

296 PAPAPAPAPAPAPAPPKE were identified as potential markers of 9 months of curing

297 [2]. The quantification of biomarkers can also be used to follow the time course of 298 ripening, being helpful in product development and process optimisation to ensure the

299 quality and safety of dry-cured ham [38]. In this regard, two peptides were detected as

300 potential candidates to be biomarkers of the manufacturing process of Norwegian dry-

301 cured hams [54]. Table 2 shows the sequences of peptides identified as potential

302 biomarkers of the dry-cured ham processing.

303 The final quality of dry-cured hams also depends on the raw material used in its

304 preparation as differences in pig genotypes result in different action of endogenous

305 enzymes and thus different peptide profiles. In this regard, peptidomics was used to

306 determine peptides derived from proteins myosin light chain 1, creatine kinase,

307 myoglobin, troponin $\mathrm{T}$, and myosin heavy chain 7 that were mainly responsible for

308 quantitative differences between dry-cured hams coming from pigs of three different

309 genetic selections [4]. Moreover, differences in proteolysis among dry-cured hams of

310 different genetics and processing conditions could be assessed through this approach. A

311 study comparing peptide profiles of Iberian dry-cured ham processed for 24 months and

312 traditional Spanish dry-cured ham of 14 months showed that the most influential peptides

313 were derived from creatine kinase, actin, troponin $\mathrm{T}$, and myosin heavy and light chains

314 [43]. In the case of different types of European dry-cured hams, peptides from myosin

315 light chain, glyceraldehyde-3-phosphate dehydrogenase, myosin heavy chain, and 
316 myosin 4 were reported to be responsible for differences between Spanish Teruel, Italian

317 Parma and Belgian hams [47].

\section{$318 \quad 4.3$ Analysis of post-translational modifications in peptides}

319 Peptidomic approaches are useful tools for detecting post-translational modifications

320 (PTM) such as oxidation, deamidation, phosphorylation, and carbamylation, which can

321 modify physical and chemical properties of proteins and peptides. Novel advances in MS

322 have permitted the analysis of PTM despite the existing difficulties related to the 323 complexity of the involved mechanisms, wide variety of generated products as well as

324 the low abundance and lability of some of them under the conditions of sample 325 preparation and/or analysis. Tandem MS techniques allow the determination of the site 326 and nature of PTM because modified amino acids are mass-shifted and thus identifiable 327 in the fragmentation pattern. In addition, the combination of MS/MS with LC can be 328 employed for the quantification of such modifications [62].

329 Oxidative processes play an important role in the quality and sensory characteristics of 330 dry-cured hams. Several peptides showing methionine oxidation derived from different 331 myofibrillar proteins such as titin, myosin, and nebulin, among others, have been 332 identified in Spanish dry-cured hams [63]. Moreover, a recent work has studied the evolution of oxidised peptides during the processing of 9 months dry-cured ham, identifying and quantifying peptides with methionine, proline, and tryptophan oxidations, and showing quantitative differences resulting from specific processing conditions [64].

336 An example of relative quantification of peptides with oxidised methionine is shown in

337 Table 3. Also peptide deamidation, mainly of asparagine and glutamine residues, in 12 months dry-cured hams has been reported to be influenced by processing conditions like temperature, $\mathrm{pH}$, and salting of hams [65]. These studies provide valuable information

340 about PTM mechanisms and their effects at peptide level during the dry-cured ham 
341 processing, which could affect the nutritional and quality characteristics of the final 342 product.

\section{5. Current tendencies and future perspectives}

345 Further improvements in mass spectrometers and novel MS techniques are permitting a

346 significant advance in the field of peptidomics through the use of empirical approaches

347 for the identification and quantification of peptides. However, bioinformatics approaches 348 based on the combination of computational tools and peptide databases are gaining 349 importance in recent years as a time- and cost-effective strategy to study the 350 characteristics of peptides, predict proteolysis and biological activities, and evaluate 351 proteins as potential precursors $[41,66]$. In this regard, in silico analysis including 352 quantitative structure-activity relationship models (QSAR), molecular docking simulations and chemometrics are being widely used for bioactive peptide discovery, allowing a greater knowledge of their characteristics and mechanisms of action as well as the optimisation of proteolysis variables when searching for a specific activity $[67,68]$.

356 Additionally, current tendencies in the study of bioactive peptides are related to their

357 bioavailability as more information regarding peptide changes in human body during 358 digestion, transport, absorption, and metabolism, as well as final available concentrations 359 are necessary to better understand their effects in the organism. In fact, the amount of 360 active peptide that is ingested and is able to reach its target site will determine its effect 361 and impact in the human system, thus quantification of bioactive peptides is needed in

362 future bioavailability studies. Nevertheless, there are still several challenges for 363 determining the content of bioactive peptides in blood stream and target organs as the low 364 concentration levels make instruments to be in the limits of their range of action [37].

365 Moreover, the difficulty in the analysis is also due to the complexity of biological matrices, 
366 whose main composition is protein, as well as peptide-matrix interactions that can lead to

367 structural changes in bioactive peptides affecting their release, bioavailability, and 368 bioactivity [69].

369 The high efficiency of peptidomic strategies combined with the predictive capability of 370 bioinformatics approaches represents a powerful tool for the discovery and analysis of 371 bioactive peptides as well as for a better understanding of proteolysis phenomena, which 372 could be applied in future studies towards the improvement of the final quality and the 373 development of added-value processed meat products.

374

\section{Acknowledgements}

376 Grant AGL2014-57367-R and FEDER funds from the Spanish Ministry of Economy,

377 Industry and Competitiveness are acknowledged. Ramón y Cajal postdoctoral contract to 378 LM is also acknowledged.

\section{Conflicts of interest}

381 Authors declare that none of them have any conflict of interest.

382

383

\section{References}

384 [1] S. Sforza, V. Cavatorta, F. Lambertini, G. Galaverna, A. Dossena, R. Marchelli, J. 385 Dairy Sci. 2012, 95, 3514.

386 [2] M. Gallego, L. Mora, F. Toldrá, J. Proteomics 2016, 147, 98.

387 [3] M. Gallego, L. Mora, M. Hayes, M. Reig, F. Toldrá, Food Res. Int. 2017, 97, 296.

388 [4] L. Mora, L. Calvo, E. Escudero, F. Toldrá, Food Res. Int. 2016, 86, 74. 
[5] F. Toldrá, in Food Biochemistry and Food Processing (Eds: B. K. Simpson, L. M. L. Nollet, F. Toldrá, S. Benjakul, G. Paliyath, Y. H. Hui), Wiley-Blackwell, Ames, IA 2012, Ch. 16.

[6] A. Di Luccia, G. Picariello, G. Cacace, A. Scaloni, M. Faccia, V. Liuzzi, G. Alviti, S. S. Musso, Meat Sci. 2005, 69, 479.

[7] E. Rodríguez-Nuñez, M. C. Aristoy, F. Toldrá, Food Chem. 1995, 53, 187.

[8] L. Théron, T. Sayd, J. Pinguet, C. Chambon, N. Robert, V. Santé-Lhoutellier, Meat Sci. 2011, 88, 82 .

[9] G. Picariello, A. De Martino, G. Mamone, P. Ferranti, F. Addeo, M. Faccia, S. S. Musso, A. Di Luccia, J. Chromatogr. B Analyt. Technol. Biomed. Life Sci. 2006, 833, 101.

[10] D. Castejon, P. Villa, M. M. Calvo, G. Santa-Maria, M. Herraiz, A. Herrera, Magn. Reson. Chem. 2010, 48, 693.

[11] F. Bajd, M. Škrlep, M. Čandek-Potokar, J. Vidmar, I. Serša, I. Meat Sci. 2016, 122, 109.

[12] A. Fabbro, M. Bencivenni, E. Piasentier, S. Sforza, M. L. Stecchini, G. Lippe, Eur Food Res. Technol. 2016, 242, 881.

[13] M. Gallego, L. Mora, M. C. Aristoy, F. Toldrá, Food Chem. 2014, 196, 437.

[14] G. Paredi, R. Benoni, G. Pighini, L. Ronda, A. Dowle, D. Ashford, J. Thomas, G.

Saccani, R. Virgili, A. Mozzarelli, J. Agric. Food Chem. 2017, 65, 6307.

[15] L. Mora, F. Toldrá, in Advanced Technologies for Meat Processing (Eds: F. Toldrá, L. M. L. Nollet), Taylor \& Francis, Boca Ratón, FL 2017, Ch. 17.

[16] L. Mora, P. D. Fraser, F. Toldrá, Food Res. Int. 2013, 54, 1292.

[17] M. A. Sentandreu, M. Armenteros, J. J. Calvete, A. Ouali, M. C. Aristoy, F. Toldrá, J. Agric. Food Chem. 2007, 55, 3613. 
414 [18] L. Mora, M. A. Sentandreu, K. M. Koistinen, P. D. Fraser, F. Toldrá, P. M. Bramley, 415 J. Agric. Food Chem. 2009, 57, 3228.

416 [19] L. Mora, M. A. Sentandreu, F. Toldrá, J. Agric. Food Chem. 2011, 59, 3884.

417 [20] L. Mora, M. A. Sentandreu, F. Toldrá, Food Chem. 2010, 123, 691.

418 [21] L. Mora, M. Gallego, E. Escudero, M. Reig, M. C. Aristoy, F. Toldrá, Int. J. Food 419 Microb. 2015, 212, 9.

420 [22] M. Gallego, L. Mora, M. C. Aristoy, F. Toldrá, Food Chem. 2015, 167, 326.

421 [23] M. Gallego, L. Mora, M. C. Aristoy, F. Toldrá, Food Chem. 2014, 149, 121.

422

[24] L. Mora, M. A. Sentandreu, P. D. Fraser, F. Toldrá, P. M. Bramley, J. Agric. Food 423 Chem. 2009, 57, 8982.

424 [25] L. Mora, M. L, Valero, M. M. Sánchez del Pino, M. A. Sentandreu, F. Toldrá, J. 425 Proteomics 2011, 74, 442.

426 [26] L. Mora, F. Toldrá, P. M. Bramley, Food Technol. Biotechnol. 2012, 50, 343.

427 [27] L. Mora, M. Gallego, M. C. Aristoy, P. D. Fraser, F. Toldrá, Food Control 2015, 48, 428102.

429 [28] C. Z. Zhu, W. Tian, M. Y. Li, Y. X. Liu, G. M. Zhao, Int. J. Food Prop. 2017, 20, 430 S2980.

431 [29] F. Toldrá, M. Flores, Crit. Rev. Food Sci. Nutr. 1998, 38, 331.

432 [30] F. Toldrá, M. C. Aristoy, M. Flores, Food Res. Int. 2000, 33, 181.

433 [31] M. A. Sentandreu, S. Stoeva, M. C. Aristoy, K. Laib, W. Voelter, F. Toldrá, J. Food 434 Sci. 2003, 68, 64.

435 [32] Y. Dang, X. Gao, F. Ma, X. Wu, LWT - Food Sci. Technol. 2015, 60, 1179.

436 [33] M. Bantscheff, M. Schirle, G. Sweetman, J. Rick, B. Kuster, Anal. Bioanal. Chem. $437 \quad 2010,389,1017$.

438 [34] W. Zhu, J. V. Smith, C. M. Huang, J. Biomed. Biotechnol. 2010, 840518. 
[35] C. A. Sherwood, A. Eastham, L. W. Lee, J. Risler, H. Mirzaei, J. A. Falkner, D. B.

440 Martin, J. Proteome Res. 2009, 8, 3746.

441 [36] P. Picotti, R. Aebersold, Nat. Methods 2015, 9, 555.

442 [37] L. Mora, M. Gallego, M. Reig, F. Toldrá, Trends Food Sci. Technol. 2017, 69, 306.

443 [38] K. F. Degnes, H. F. N. Kvitvang, H. Haslene-Hox, I. M. Aasen, Food Bioprocess 444 Technol. 2017, 10, 1122.

445 [39] M. Gallego, L. Mora, F. Toldrá, Food Chem. 2018, 258, 8.

446 [40] W. Jira, A. Sadeghi-Mehr, D. A. Brüggemann, F. Schwägele, Meat Sci. 2017, 129, 44781.

448 [41] L. Sánchez-Rivera, D. Martínez-Maqueda, E. Cruz- Huerta, B. Miralles, I. Recio, 449 Food Res. Int. 2014, 63, 170.

450 [42] E. Escudero, M. C. Aristoy, H. Nishimura, K. Arihara, F. Toldrá, Meat Sci. 2012, 91, 451306.

452 [43] L. Mora, E. Escudero, K. Arihara, F. Toldrá, Food Res. Int. 2015, 78, 71.

453 [44] E. Escudero, L. Mora, F. Toldrá, Food Chem. 2014, 161, 305.

454 [45] E. Escudero, L. Mora, P. D. Fraser, M. C. Aristoy, K. Arihara, F. Toldrá, J. 455 Proteomics 2013, 78, 499.

456 [46] L. Dellafiora, S. Paolella, C. Dall'Asta, A. Dossena, P. Cozzini, G. Galaverna, J. 457 Agric. Food Chem. 2015, 63, 6366.

458 [47] L. Mora, E. Escudero, F. Toldrá, Food Res. Int. 2016, 89, 638.

459 [48] E. Escudero, L. Mora, P. D. Fraser, M. C. Aristoy, F. Toldrá, Food Chem. 2013, 138, $460 \quad 1282$.

461 [49] L. Mora, E. Escudero, P. D. Fraser, M. C. Aristoy, F. Toldrá, Food Res. Int. 2014, $46256,68$.

463 [50] C. Z. Zhu, W. G. Zhang, G. H. Zhou, X. L. Xu, J. Sci. Food Agric. 2016, 96, 99. 
464 [51] L. J. Xing, Y. Y. Hu, H. Y. Hu, Q. F. Ge, G. H. Zhou, W. G. Zhang, Food Chem. 465 2016, 194, 951.

466 [52] P. Castellano, L. Mora, E. Escudero, G. Vignolo, R. Aznar, F. Toldrá, Food 467 Microbiol. 2016, 59, 133.

468 [53] M. Gallego, M. C. Aristoy, F. Toldrá, Meat Sci. 2014, 96, 757.

469 [54] I. Petrova, I. Tolstorebrov, L. Mora, F. Toldrá, T. M. Eikevik, Meat Sci. 2016, 121, 470243.

471 [55] C. Z. Zhu, W. G. Zhang, G. H. Zhou, X. L. Xu, Z. L. Kang, Y. Yin, J. Agric. Food 472 Chem. 2013, 61, 1265.

473 [56] S. Paolella, C. Falavigna, A. Faccini, R. Virgili, S. Sforza, C. Dall'Asta, A. Dossena, 474 G. Galaverna, Food Res. Int. 2015, 67, 136.

475 [57] C. Z. Zhu, W. G. Zhang, Z. L. Kang, G. H. Zhou, X. L. Xu, Meat Sci. 2014, 96, 783. 476 [58] M. Gallego, L. Mora, M. Reig, F. Toldrá, Food Res. Int. 2018, 105, 873.

477 [59] M. Gallego, C. Grootaert, L. Mora, M. C. Aristoy, J. Van Camp, F. Toldrá, J. Funct. $478 \quad$ Foods 2016, 21, 388.

479 [60] S. M. Martínez-Sánchez, A. Minguela, D. Prieto-Merino, M. P. Zafrilla-Rentero, J. 480 Abellán-Alemán, S. Montoro-García, Nutrients 2017, 9, 321.

481 [61] S. Montoro-García, M. P. Zafrilla-Rentero, F. M. Celdrán-de Haro, J. J. Piñero-de 482 Armas, F. Toldrá, L. Tejada-Portero, J. Abellán-Alemán, J. Funct. Foods 2017, 38, 160. 483 [62] A. M. N. Silva, R. Vitorino, M. R. M. Domingues, C. M. Spickett, P. Domingues, $484 \quad$ Free Radic. Biol. Med. 2013, 65, 925.

485 [63] M. Gallego, L. Mora, M. C. Aristoy, F. Toldrá, Food Chem. 2015, 187, 230.

486 [64] M. Gallego, L. Mora, F. Toldrá, Food Chem. 2018, 239, 823.

487 [65] M. Cañete, L. Mora, F. Toldrá, Food Chem. 2017, 229, 710. 
488 [66] D. Agyei, C. M. Ongkudon, C. Y. Wei, A. S. Chan, M. K. Danquah, Anal. Bioanal.

489 Chem. 2018, 1.

490 [67] J. Carrasco-Castilla, A. J. Hernández-Álvarez, C. Jiménez-Martínez, G. F. Gutiérrez-

491 López, G. Dávila-Ortiz, Food Eng. Rev. 2012, 4, 224.

492 [68] A. B. Nongonierma, R. J. FitzGerald RJ, RSC Adv. 2016, 6, 75400.

493 [69] C. C. Udenigwe, V. Fogliano, J. Funct. Foods 2017, 35, 9.

494 
496 Figure 1. Scheme showing the applications of peptidomics in dry-cured ham.

497

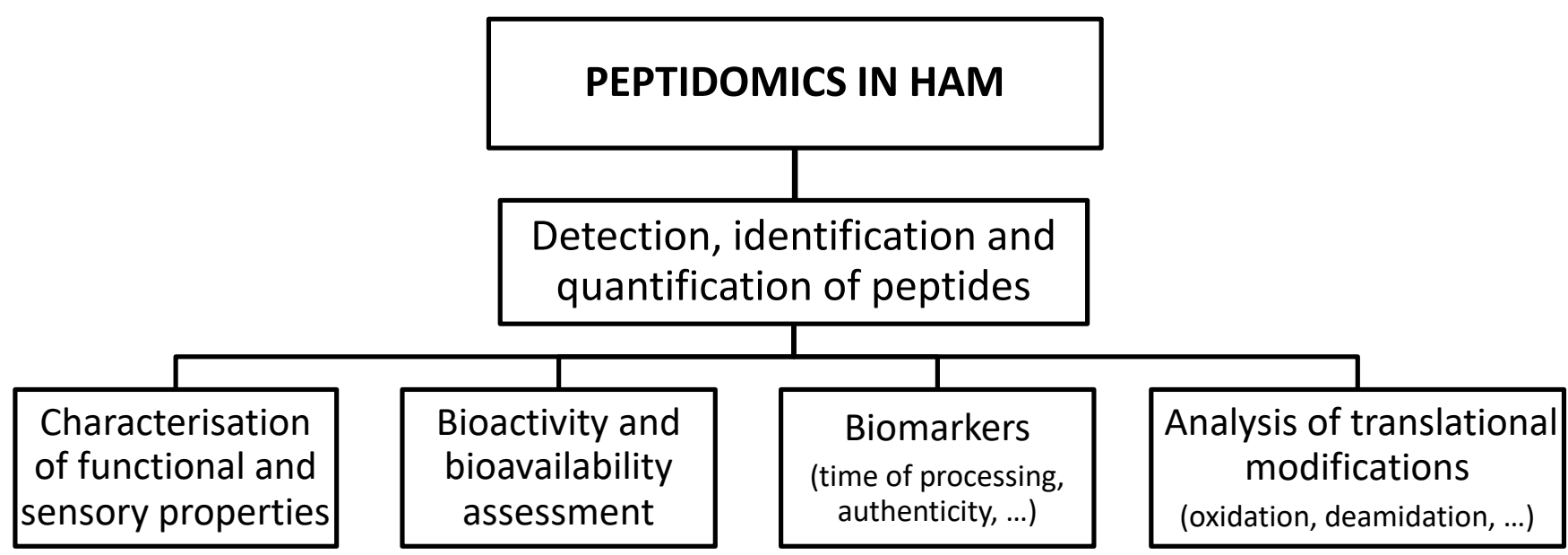

498

499

500

501

502

$503 \quad$ Figure 1.

504 